\title{
Immunohistochemistry in district general hospitals in England and Wales
}

\author{
P A HALL, ${ }^{*} \dagger$ PAOLA DOMIZIO,* G SLAVIN, $\dagger$ D A LEVISON† \\ From the $\dagger$ Department of Histopathology, and ${ }^{*}$ Imperial Cancer Research Fund Department of Medical \\ Oncology, St Bartholomew's Hospital, London
}

SUMMARY The findings of a survey on the use of immunohistochemistry in district general hospitals in England and Wales are reported. Immunohistochemistry is used in most district hospitals, contributes to the accuracy and objectivity of some histopathological diagnoses, and is considered to be generally useful though not without drawbacks. Its expansion is being hindered by lack of funds for reagents and staff. In a few regions attempts are being made to rationalise expenditure and coordinate development of the service. We believe that if this can be done at a regional or national level then the relatively small cost entailed will be justified by a resulting improvement in the quality of patient care.

Immunohistochemistry is the technique of showing the presence of antigens in histological sections by means of labelled antibodies. Its use has been advocated to increase the accuracy and objectivity of histopathological diagnosis, which can be vital clinically, particularly in the diagnosis of tumours which have hitherto posed particular problems - for example, lymphomas and anaplastic carcinomas. ${ }^{1}$ Diagnosis of these may now be achieved more reproducibly with the help of immunohistochemistry. Immunohistochemistry helps to distinguish mesothelioma from adenocarcinoma ${ }^{2}$ and can indicate the prostatic origin of a bony metastasis. ${ }^{3}$ The demonstration of a variety of tumour markers is also of clinical importance..$^{3}$ In addition to its importance in the investigation of neoplastic disorders, immunohistochemistry is now an integral part of the examination of skin and renal biopsy specimens. ${ }^{45}$

Early experience with immunohistochemistry was marred by the often poor quality of polyclonal heteroantisera, the reliance on immunofluorescence, and often the need for frozen sections. The introduction of immunoenzyme methods applicable to paraffin sections, coupled with the development of hybridoma technology ${ }^{6}$ and ready availability of monoclonal antibodies, led to a rapid increase in the use of immunohistochemical techniques.

Evidence of this rapid expansion is provided by the figure which shows the pronounced increase in immu-

Accepted for publication 11 May 1987 nohistochemically based published reports in Britain. Most of these reports are from teaching centres and research institutions. It has not been clear, however, whether increased use of immunohistochemistry has also occurred in district general hospitals where a major proportion of diagnostic histopathology takes place. We therefore undertook a survey of district general hospital histopathology laboratories in England and Wales in an attempt to define their use of immunohistochemistry, to assess pathologists' attitudes towards the technique, and to identify any factors that might be impeding its use and expansion.

\section{Methods}

The questionnaire sought information on the practice of immunohistochemistry, covering start date, frequency of use, changing use, attitudes to expansion and relevant factors governing that expansion, effects on other laboratory practices and diagnosis, and preferential use of technical data and antibodies. We also invited other comments. The questionnaire was piloted within our department and also to a small number of pathologists in district general hospitals. It was distributed to 189 pathologists in 189 such hospitals throughout England and Wales in early November 1986, together with an explanatory letter. Names were randomly selected from a database held by the Royal College of Pathologists. Nine were subsequently deleted because: they were part of a teaching hospital group $(n=3)$; they were specialist hospitals and not district general hospitals $(n=4)$, or 


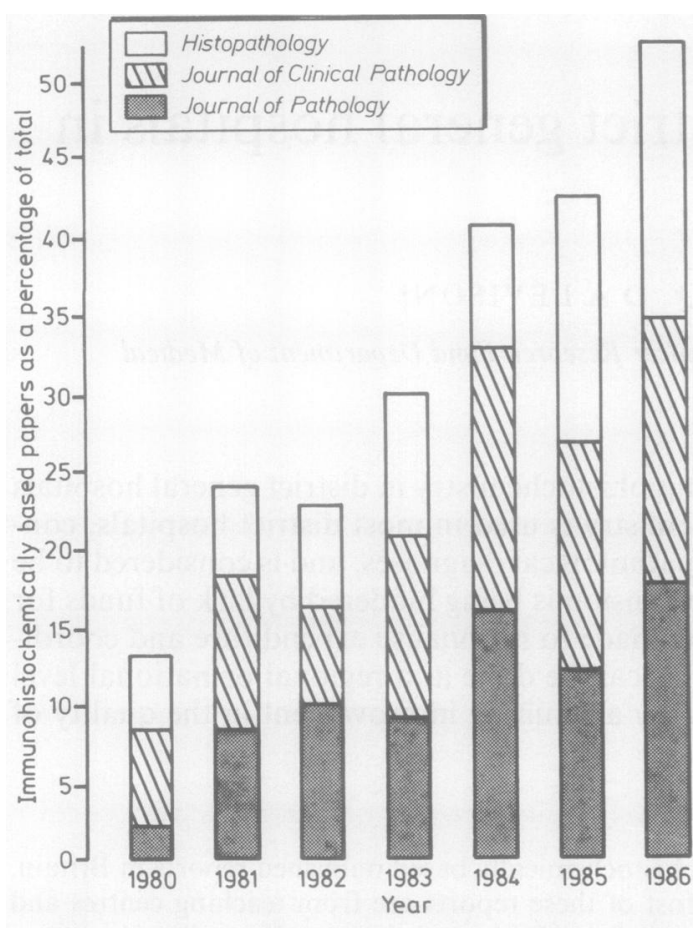

Percentage of immunohistochemically based articles in three British pathology journals. $100 \%=$ the sum of all histopathology articles in all three journals.

were hospitals with centralised pathology services $(\mathrm{n}=2)$.

\section{Results}

One hundred and forty seven replies were received from a possible $180(81.7 \%$ response $)$ and 104 laboratories $(70.7 \%)$ were found to be performing immunohistochemistry. A few respondents failed to answer all questions.

\section{THE USE OF IMMUNOHISTOCHEMISTRY \\ When it was introduced}

Though immunofluorescence techniques have been in widespread use for many years to show the presence of serum autoantibodies, tissue immunohistochemistry was being used in only eight departments prior to 1976 and was only established in 19 laboratories by the end of 1979. By 1981 this had increased to 48 and by 1986104 district general hospitals were using these techniques to some extent. The rise in use of immunohistochemistry in district general hospitals closely reflects the proportion of publications devoted to this subject.
How much is done

The amount of immunohistochemistry performed in individual laboratories varied. Sixteen departments apply the method to one case about once a month or less; 28 use immunohistochemistry once a week; while a further 60 use it more frequently, and 19 of these five times per week or more. Eighty nine respondents stated that their use of immunohistochemistry was increasing, 77 slowly and 12 rapidly. No change in use was reported by 14 respondents, and one laboratory reported a decrease. Eighty five departments expressed a desire to increase further the use of immunohistochemistry. Thirteen were happy with their current level of usage.

\section{Antibodies used and tissues examined}

The number of antibodies used by different laboratories also varied. Thirty four laboratories used 10 or fewer antibodies; 19 used more than 20 antibodies; 51 used between 11 and 20 .

A wide range of types of polyclonal and monoclonal antibodies are used. Table 1 shows the most popular reagents. Although many respondents felt that anti-immunoglobulin antibodies were difficult to interpret, these were used by nearly all departments; 31 respondents described them as among their five most useful antibodies. Antileucocyte common antigen and antibodies to cytokeratins or epithelial mem? brane antigen, or both, were also extensively used: 6 respondents reported these reagents in their list of five most useful antibodies.

Fourteen departments phenotyped lymphomas but only four of these did so in detail. Renal biopsy specimens were examined by 34 departments and skin

Table 1 Antibodies most used in 104 departments performing immunohistochemistry

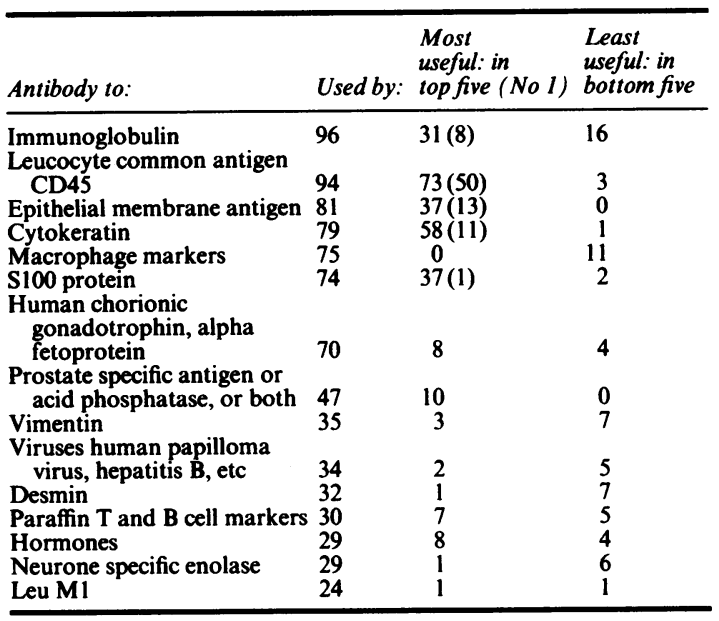


biopsy specimens by 67 . Cytological preparations were examined using immunohistochemical methods in 35 laboratories. Research projects using immunohistochemistry were undertaken by 39 departments.

\section{Techniques used}

The peroxidase-antiperoxidase method (PAP) was the most popular, being used by 85 departments, with the indirect immunoperoxidase method being used by 58 . Immunofluorescence is still extensively used ( 54 laboratories), particularly for the examination of skin biopsy specimens. The newer methods such as avidin-biotin complex (ABC), alkaline phosphataseantialkaline phosphatase (APAAP), and immunogold silver (IGSS) are much less extensively used $(24,15$, and 14 laboratories, respectively), presumably due to their relative complexity and expense. A surprising number of departments had more than one method available: 47 departments used three or more methods and seven departments used five or more methods.

\section{Effects on laboratory practice and diagnosis}

It might be expected that the introduction of immunohistochemical methods would lead to a reduction in the use of conventional special stains, but 83 of 101 respondents reported no change in their use of special stains, and in nine of the 18 laboratories where there had been a fall, this had been by $10 \%$ or less. In only two departments had there been a fall of more than $50 \%$.

It is difficult to assess the contribution to diagnosis of a new method such as immunohistochemistry as there are no objective reference standards or suitable performance indicators. We have found, however, that cases may be divided into those where immunohistochemistry makes a major contribution-that is, it establishes or confirms a diagnosis-those where it makes a minor contribution-that is, it provides some contributory or supportive information-those where it makes no contribution, and those where it actually confuses the diagnosis. Table 2 summarises the views of the respondents on the contribution of immunohistochemistry using such a scheme of appraisal.

\section{Interpretation of immunohistochemical techniques}

In our experience the interpretation of immunohistochemical preparations is often difficult and interestingly only 20 of 101 respondents shared this view. Sixty four felt that interpretation was sometimes difficult and 17 felt that interpretation was usually easy. Fifty eight respondents specified the antibodies that give them most difficulty. The most commonly cited were anti-immunoglobulins in the context of lymphoproliferative disease where $46 \%$ of the 58 had difficulty.
Table 2 Contribution of immunohistochemistry to diagnosis expressed as percentage

\begin{tabular}{lllll}
\hline Cases & $\begin{array}{l}\text { Major } \\
\text { contribution } \\
(n=100)\end{array}$ & $\begin{array}{l}\text { Minor } \\
\text { contribution } \\
(n=96)\end{array}$ & $\begin{array}{l}\text { Nil } \\
(n=85)\end{array}$ & $\begin{array}{l}\text { Confuses } \\
\text { diagnosis } \\
(n=77)\end{array}$ \\
\hline All & 2 & 0 & 0 & 0 \\
Many & 30 & 21 & 12 & 5 \\
Some & 38 & 59 & 34 & 21 \\
Few & 28 & 19 & 42 & 61 \\
None & 2 & 1 & 12 & 13 \\
\hline
\end{tabular}

\section{Advice from the companies}

Eighty respondents stated that the commercial suppliers of immunohistochemical reagents gave useful and helpful advice and support, but 19 felt that these companies were sometimes overoptimistic, misleading, or insufficiently critical.

\section{LIMITATIONS ON THE USE OF}

IMMUNOHISTOCHEMISTRY

\section{Laboratories not performing immunohistochemistry}

Forty three laboratories (29.3\%) replied that they were not using immunohistochemistry. Of those, 15 intended to begin in 1987, a further four were hoping to start in 1988, and two were hoping to begin "sometime". Twenty two laboratories did not intend to provide an immunohistochemical service.

In many laboratories the lack of funds $(n=2)$ or technical staff $(n=29)$ had prevented development of a service and these shortages often occur together $(n=17)$. One respondent stopped using immunohistochemistry in 1983 due to lack of funds. Lack of time or lack of experience on the part of the pathologist is also important $(n=15)$, and two respondents stated that they were awaiting the appointment of a second consultant before starting. Only a few departments failed to provide a service on technical grounds: two pathologists had doubts about the methodology while four saw no advantage in the technique over conventional histology.

\section{Laboratories performing immunohistochemistry}

Lack of suitable MLSO staff was reported by 43 departments, and funds by 53 , as major factors impeding expansion, while 32 departments considered both factors to be important. Lack of experience on the part of the pathologist (13 departments), lack of suitable space (11 departments), and lack of time (eight departments) were other important restrictive factors. Twenty two laboratories gave no reason for not expanding while one respondent stated that expansion of immunohistochemistry was contrary to district policy. 


\section{Discussion}

Most histopathological diagnoses of both neoplastic and non-neoplastic conditions can be made without resort to the application of immunohistochemical methods. Goudie estimated that immunohistochemistry was performed in $3 \%$ of cases in one major hospital $^{7}$ but this figure excluded renal and skin biopsy specimens and is thus likely to be an underestimate. In our department in 1986 immunohistochemistry was applied to $5 \%$ of all cases, but such figures are strongly influenced by the nature of the pathologist's workload and interests and the availability of antibodies. In certain situations, however, immunohistochemistry does provide a more objective and reproducible means of making diagnoses than was previously possible and this is of increasing clinical importance with expanding therapeutic options. ${ }^{8}$ As table 2 illustrates, $68 \%$ of respondents felt that immunohistochemistry made a major contribution in some or many cases.

The diagnostic limitations of the technique are real; it is not a magic wand. It demands technical skill and care for consistent and interpretable results. It also requires experience on the part of the pathologist in the selection of appropriate antibodies and in the interpretation of the results of staining. When considering the application of tumour markers it must be remembered that there are very few, if any, truly specific markers, and careful interpretation and correlation of the results with the clinical findings is required. With increasing experience it is apparent that a number of antigens previously felt to be specific for certain tumours can be expressed in others. For example, Gatter et al have shown that some epithelial tumours express the "connective tissue marker" vimentin and the "muscle antigen" desmin, thus illustrating pitfalls that may occur from overreliance on this technique. ${ }^{9}$ Panels of antibodies rather than a single marker are often of great value. Such limitations as these are no doubt reflected by the notion that immunohistochemistry was felt to confuse diagnosis in a few cases by $61 \%$ of respondents (table 2).

This study has shown that immunohistochemistry is now widely used in the histopathology departments of district general hospitals throughout England and Wales. There are, however, problems with its expansion and continued use. In particular, the lack of resources of all types is an important factor. There is a need for sufficient medical staff within a department to provide the drive to apply the technique within the laboratory. In those laboratories where the staffing levels fall below those recommended by the Royal College of Pathologists it is difficult to envisage how even the most enthusiastic pathologist could find the time to develop a service which needs critical control and constant evaluation. In addition, suitable numbers of skilled MLSO staff are required to support the medical staff. A deficiency of MLSO's was the major factor in impeding development in those departments not yet performing immunohistochemistry and nearly half of those where it has been established. Recurrent costs also need to be adequately provided for as the antibodies are often expensive.

The need for some kind of "best buy" guide was suggested by 28 respondents. The currently available antibodies are often expensive and there are sometimes several companies making similar reagents. Very few laboratories have the time or expertise to assess systematically the merits of such reagents and a regional or national system of antibody screening could be of potential value. A national database of immunohistochemical information was suggested by 34 respondents as being of possible use, disseminating data on the suitability of reagents, various technical aspects, and on the false positive and false negative rates for various antibodies in specific applications.

During the course of this study we found that within a few regions there are established or planned coordinated policies on the subject of immunohistochemistry. These entail the referral of sections to specialist centres for certain, less commonly required tests, the sharing of antibodies between hospitals, and usually the performance of commonly required test 8 by individual hospitals. We believe that this is a sen sible approach to the planning and development immunohistochemical services in this country.

Most of our colleagues believe, according to the results of our survey, that immunohistochemical methods that are relatively easy to apply and are of clinical importance-for example, the distinction of carcinoma from lymphoma-should be available in district general hospital laboratories. Performing such tests at the local level avoids delays in reporting and maintains the interest of all laboratory staff. Undoubtedly the staff and reagents required to ? provide immunohistochemistry in all district general hospitals will add to the cost of histopathology, but if the development can be coordinated at a regional or $\delta$ national level then the cost will be justified by the $₹$ resulting improvement in the quality of patient care.

We thank Drs AJ d'Ardenne and Tim Spector for $\overparen{\Omega}$ helpful discussion during this study. We are grateful $\tilde{O}$ to Mrs Jeanette Turner for helping with adminis- N trative and secretarial aspects of the study and to The 0 Royal College of Pathologists for permission to use

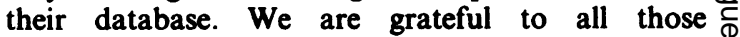
histopathologists who spared the time to complete $\stackrel{\oplus}{+}$ and return our questionnaire. 


\section{References}

1 Gatter KC, Alcock C, Heryet A, Mason DY. Clinical importance of analysing tumours of uncertain origin with immunohistochemical techniques. Lancet 1985;i:1302-5.

2 Ghosh AK, Gatter KC, Dunnill MS, Mason DY. Immunohistological staining of reactive mesothelium, mesothelioma and lung carcinoma with a panel of monoclonal antibodies. J Clin Pathol 1987;40:19-25.

3 Heyderman E. The immunoperoxidase technique in histopathology with special reference to malignant tumours of epithelium and germ cell origin. University of London (Thesis) 1983.

4 Chu AC. Immunocytochemistry in dermatology. In: Polak JM, Van Noorden S, eds. Immunocytochemistry, modern methods and applications. 2nd ed. Bristol: Wright PSG, 1986:618-37.

5 Evans DJ. Immunohistology in the diagnosis of glomerular disease. In: Polak JM, Van Noorden S, eds. Immunocytochemistry, modern methods and applications. 2nd ed. Bristol:
Wright PSG, 1986:638-49.

6 Köhler M, Milstein C. Continuous culture of fused cells secreting antibody of predefined specificity. Nature 1975;256:495-7.

7 Goudie RB. Some implications of immunocytochemistry for routine diagnostic histopathology. In: Polak JM, Van Noorden S, eds. Immunocytochemistry, modern methods and applications. 2nd ed. Bristol: Wright PSG, 1986:674-81.

8 d'Ardenne AJ, Butler MG, Hall PA, Stearn PM. The role of immunocytochemistry in routine tumour diagnosis. $J$ Pathol 1986;149:312A.

9 Gatter KC, Dunnill MS, van Muijen DNP, Mason DY. Coexpression of intermediate filaments in human lung tumours. $J$ Clin Pathol 1986;39:950-4.

Requests for reprints to: Dr Peter A Hall, Department of Histopathology, St Bartholomew's Hospital, West Smithfield, London EC1A 7BE, England. 